\title{
Tf-lipoplexes for neuronal siRNA delivery: A promising system to mediate gene silencing in the CNS
}

\author{
A.L.C. Cardoso $^{\text {a,b }}$, S. Simões ${ }^{\text {a,c }}$, L.P. de Almeida ${ }^{a, c}$, N. Plesnila ${ }^{\text {d }}$, M.C. Pedroso de Lima ${ }^{a, b, *}$, \\ E. Wagner ${ }^{\mathrm{e}}$, C. Culmsee $\mathrm{e}^{\mathrm{e} 1}$ \\ a Center for Neuroscience and Cell Biology, University of Coimbra, 3004-517 Coimbra, Portugal \\ b Department of Biochemistry, Faculty of Science and Technology, University of Coimbra, Apartado 3126, 3001-401 Coimbra, Portugal \\ c Laboratory of Pharmaceutical Technology, Faculty of Pharmacy, University of Coimbra, 3000-295 Coimbra, Portugal \\ ${ }^{\mathrm{d}}$ Department of Neurosurgery E Institute for Surgical Research, University of Munich Medical Center - Großhadern, Ludwig-Maximilians University, Munich, Germany \\ e Department of Pharmacy, Ludwig-Maximilians University, Munich, Germany
}

\section{A R T I C L E I N F O}

\section{Article history:}

Received 5 May 2008

Accepted 22 August 2008

Available online 2 September 2008

\section{Keywords:}

Cationic liposomes

Transferring

SiRNA

Gene silencing

Brain

In vivo

\begin{abstract}
A B S T R A C T
Although RNAi-based gene silencing holds a great potential for treatment of neurological disorders, its application to the CNS has been restricted by low levels of tissue distribution and cellular uptake. In this work we report that cationic lipid-based vectors can enhance siRNA delivery to neurons both in vitro and in vivo. DOTAP:Chol liposomes associated with transferrin (Tf) and complexed with siRNAs (Tf-lipoplexes) were delivered to primary cultures of luciferase-expressing cortical neurons. Confocal microscopy studies revealed efficient cellular uptake of Cy3-labelled siRNAs after Tf-lipoplex delivery, which was reduced but not completely inhibited by blocking the Tf-receptor with excess Tf. Gene silencing was also evaluated after delivery of anti-luciferase or anti-c-Jun siRNAs. Our results demonstrate that Tf-lipoplexes achieve up to 50\% luciferase and c-Jun knockdown, $48 \mathrm{~h}$ after transfection, without significant cytotoxicity. Similar results were observed in vivo, where a $40 \%$ reduction of luciferase activity was found in the striatum of luciferase mice. In addition, fluorescence microscopy studies showed extensive local distribution and internalization of Tflipoplex-associated Cy3-siRNAs without tissue toxicity. Overall, our results demonstrate that Tf-lipoplexes can mediate efficient gene silencing in neuronal cells, both in vitro an in vivo, which may prove useful in therapeutic approaches to neuronal protection and repair.
\end{abstract}

(c) 2008 Elsevier B.V. All rights reserved.

\section{Introduction}

The central nervous system (CNS) is the most complex and specialized organ in the human body, controlling almost all physiological processes through the action of neuronal cells. Due to their critical role, these postmitotic cells are irreplaceable and, once damaged, show little capacity for functional recovery. One of the major goals of current neuroscience research is to understand the mechanisms involved in neuronal degeneration and develop effective therapies to prevent neuronal cell death associated with neurological disorders.

Although numerous genes and toxic proteins thought to be involved in neuronal degeneration have been identified [1-3], the complexity of brain function and the crosstalk between the different

\footnotetext{
* Corresponding author. Department of Biochemistry, Faculty of Sciences and Technology, University of Coimbra, Apartado 3126, 3001-401 Coimbra, Portugal. Tel.: +351 239820 190; fax: +351239853607.

E-mail addresses: mdelima@ci.uc.pt (M.C. Pedroso de Lima), culmsee@staff.uni-marburg.de (C. Culmsee).

${ }^{1}$ Current address: Clinical Pharmacy - Pharmacology and Toxicology, Faculty of Pharmacy, Philipps-University of Marburg, Karl v. Frisch Strasse 1, 35043 Marburg, Germany. Tel.: +49 6421 25780; fax: +49 642125720 .
}

cell death and survival pathways hampers the process of attributing a specific role to a particular protein. RNA interference (RNAi) has recently emerged as a powerful tool in functional genomic studies, allowing to dissect entire signalling pathways and understand the molecular mechanisms of neurobiological processes [4], thereby facilitating rapid identification and validation of possible therapeutic targets. Moreover, RNAi holds a pronounced therapeutic potential $[5,6]$, since application of small interfering RNAs (siRNAs) may allow specific knockdown of selected toxic proteins, even when allelespecific silencing is needed, as in the case of dominantly inherited disorders $[7,8]$.

Nevertheless, the development of RNAi-based therapeutics for in vivo application faces the same challenge common to all classes of drugs: achieving an efficient and sustained distribution into the target tissue at sufficient concentrations to accomplish a therapeutic effect [9]. The CNS presents additional obstacles for drug delivery, due to the presence of the blood-brain barrier and the necessity to target specific neuronal subpopulations. Since siRNA molecules need to gain access to the interior of the cell to promote mRNA cleavage, siRNA stabilization in the extracellular and intracellular environments during the delivery process and their efficient uptake by neuronal 
cells are major challenges to a successful therapeutic application [6]. Although siRNAs are more resistant to nuclease attack than singlestranded DNA and RNA, they are still prone to RNase degradation in biological fluids. Currently, chemically modified siRNAs with increased stability in the presence of serum are already commercially available and present an improvement for siRNA delivery in vivo [10-13]. Nevertheless, such modifications fail to solve both the poor pharmacokinetic properties common to all nucleic acid-based drugs and tissue specificity [14].

In this context, cationic liposomes can help to significantly increase the siRNA's circulation time and simultaneously reduce the dose required for efficient gene silencing by enhancing siRNA stability in serum and improving cellular uptake [15]. These lipid-based systems also present a high degree of versatility that allows surface modifications which result in a significant increase of their targeting capacity and transfection efficiency [15-19]. In this regard, DOTAP:Cholesterol/DNA complexes have been widely explored by us for both in vitro $[15,17,20]$ and in vivo [16] gene therapy applications, mainly because of their capacity to mediate transfection in the presence of serum [18,21-23]. Also, transfection activity can be enhanced by the association of targeting ligands such as antibodies, peptides or proteins to the lipoplexes, which exploit the diversity of receptors existent at the surface of each cell type [19,24-27]. One useful cell-binding ligand is transferrin (Tf), an iron-transporting protein, which interacts with receptors ubiquitously expressed in various tissues [28]. Targeting transferrin receptors has been successfully applied for RNA and DNA delivery by coupling antibodies against these receptors [29,30], or transferrin to pegylated liposomes [31], or by associating the protein to cationic liposomes through electrostatic interactions [15,19,26,32]. In this regard, we and others have demonstrated that association of Tf to lipoplexes significantly enhances transfection efficiency by promoting lipoplex internalization in a large variety of cells [15-17,24,26,33-35], including neurons. In addition, we have reported that $\mathrm{Tf}$ can trigger cytoplasmic delivery of the carried nucleic acids through destabilization of the endosomal membrane under acidic conditions, thus further enhancing the transfection activity [20,24].

However, despite the widespread use of protein-associated cationic liposomes, there are still very few reports concerning their application for siRNA delivery to the CNS. We have recently reported the efficient siRNA complexation and gene silencing mediated by Tflipoplexes in glioblastoma and hepatocarcinoma cell lines [15]. In the present study, we applied the optimized Tf-lipoplexes to transfer siRNA targeting the firefly luciferase reporter gene into primary neuronal cultures obtained from transgenic NF-kB luciferase reporter mice. We also evaluated Tf-lipoplex-mediated siRNA delivery as well as luciferase and c-Jun silencing in vivo, after stereotactic injection in the striatum of the transgenic luciferase reporter mice. The results presented here demonstrate that Tf-lipoplexes can efficiently promote siRNA delivery to neuronal cells both, in vitro and in vivo, resulting in enhanced siRNA internalization and pronounced gene silencing effects, proposing a significant potential for therapeutic applications.

\section{Materials and methods}

\subsection{Materials}

The cationic lipid 1,2 dioleoyl-3(trimethylammonium)propane (DOTAP) and cholesterol (Chol) were purchased from Avanti Polar Lipids (Alabaster, AL, USA). Iron saturated human transferrin (Tf) was obtained from Sigma (St. Louis, MO, USA). The Cy3-labeled non-specific siRNA sequence was purchased from Ambion (Austin, TX, USA). The antiluciferase siRNA (luciferase Gl2 duplex) and the non-silencing siRNA used as control were obtained from Dharmacon (Lafayette, CO, USA). The anti-c-Jun siRNA (5'-AGTCATGAACCACGTTAAC-3') was obtained from Thermo Bioproducts. All other chemicals were obtained from Sigma unless stated otherwise.

\subsection{Animals}

All efforts were made to minimize suffering and the number of animals according to the guidelines of the German animal protection law and derived guidelines on the ethical use of animals. C57BL/6 mice were obtained from Charles River, Sulzfeld, Germany. NF- $\kappa$ B-luciferase-reporter mice (genetic background: $\mathrm{C} 57 \mathrm{BL} / 6$ ) were used for the measurement of NF- $\kappa B$ transcriptional activity as described previously [36]. The NF- $\kappa B$-luciferase transgene contained the firefly luciferase gene, driven by two NF- $\kappa$ B sites responding to p65/p50, p50/cRel and other dimer combinations of NF- $\kappa \mathrm{B}$ as established in previous studies $[37,38]$. All animals were kept under controlled light and environmental conditions ( $12 \mathrm{~h}$ dark/light cycle, $23 \pm 1{ }^{\circ} \mathrm{C}, 55 \pm 5 \%$ relative humidity) and had free access to food (Altromin, Germany) and water. The NF- $\kappa B$-luciferase reporter mouse strain was used because of several advantages of this transgenic mouse strain compared to other transgenic reporter mice. Luciferase protein expression can be easily quantified in various tissues including brain tissue as demonstrated previously $[36,39]$. The animals express luciferase in any cell under the control of a NF-kB binding promoter [38]. Therefore, there is a constitutive expression of luciferase in any tissue reflecting the constitutive activity of NF-kB under physiological conditions. It is not necessary to induce NF-kB or luciferase activity in these animals for the current application, since luciferase levels are low but easily detectable in all tissues without further manipulations.

\subsection{Liposome and complex preparation}

Cationic liposomes composed of DOTAP:Cholesterol (1:1 molar ratio) were prepared as previously described by Campbell [40] for in vitro application. Briefly, a mixture of $1 \mathrm{ml}$ of DOTAP and $1.5 \mathrm{ml}$ of cholesterol in chloroform (from stock solutions of $25 \mathrm{mg} / \mathrm{ml}$ DOTAP and $37.8 \mathrm{mg} / \mathrm{ml}$ cholesterol), was dried under nitrogen in order to obtain a thin lipid film. The film was dissolved in $100 \mu \mathrm{l}$ of ultrapure ethanol and the resulting ethanol solution was injected into $900 \mu \mathrm{l}$ of HBS buffer (Hepes-buffered saline solution, $20 \mathrm{mM}$ Hepes, $100 \mathrm{mM}$ $\mathrm{NaCl}, \mathrm{pH}$ 7.4) maintained continuously under vortex, employing a $250 \mu$ l Hamilton syringe. The resulting MLV (multilamellar vesicles) were sonicated briefly to obtain SUV (small unilamellar vesicles) and diluted with HBS to a final lipid concentration of $1.43 \mathrm{mM}(1 \mathrm{mg} / \mathrm{ml})$. Alternatively, for in vivo application, the dried lipid film was hydrated in $1.6 \mathrm{ml}$ of $5 \%$ HBG buffer (Hepes-glucose buffer: $5 \%$ glucose, $20 \mathrm{mM}$ Hepes, $\mathrm{pH}$ 7.4) and sonicated for $5 \mathrm{~min}$. The resulting liposomes were then extruded 21 times through two stacked polycarbonate membranes ( $50 \mathrm{~nm}$ pore diameter) and diluted in HBG buffer to a final DOTAP concentration of $22.5 \mathrm{mM}$. The liposomes were stored at $4{ }^{\circ} \mathrm{C}$ until use. The size of DOTAP:Cholesterol liposomes is approximately $120 \mathrm{~nm}$, a single and homogeneous population being observed, and the zeta potential is $+70 \mathrm{mV}$.

For the in vitro studies Tf-lipoplexes were prepared by preincubating a given volume of cationic liposomes (dependent on the desired $(+/-)$ charge ratio) with iron-saturated human transferrin (32 $\mu \mathrm{g} / \mu \mathrm{g}$ of siRNA) for $15 \mathrm{~min}$, before mixing the necessary volume of siRNA stock solution to achieve a final siRNA concentration of 50 or $100 \mathrm{nM}$ in each well. The mixture was further incubated for $30 \mathrm{~min}$, at room temperature before delivery to cortical neurons in culture. Alternatively, for in vivo administration, Tf-lipoplexes prepared at a 2/1 lipid/siRNA (+/-) charge ratio were obtained by mixing $0.8 \mu \mathrm{l} /$ animal of the liposome stock solution (22.5 mM DOTAP) with $0.5 \mu \mathrm{l} /$ animal of human Tf solution ( $192 \mathrm{mg} / \mathrm{ml}$ in HBG), followed by $15 \mathrm{~min}$ incubation prior to the addition of $3 \mu \mathrm{g} /$ animal of siRNAs. The resulting mixture was further incubated for $30 \mathrm{~min}$. All formulations were used immediately after being prepared. When mixed according to this protocol, transferrin (which exhibits a net negative charge) binds electrostactically with the positive charges of the cationic liposomes, resulting in a slight decrease in the net positive charge of the formulation but allowing further 
interaction of the liposome/transferrin complexes with the siRNA negatively charged molecules.

\subsection{Primary cell culture and siRNA delivery}

Primary mouse embryonic cortical neurons were obtained from C57/BL6 transgenic luciferase mice, at day 16 of gestation, as described previously [41]. After dissociation and centrifugation of the dissected cortices, the tissue was ressuspended in Neurobasal medium (Invitrogen, San Diego, CA, USA), enriched with 2\% (v/v) B27 supplement (Invitrogen), $2 \mathrm{mM}$ glutamine and $100 \mathrm{U} / \mathrm{ml}$ penicillin/ streptomycin (Invitrogen). Cells were plated at a $0.5 \times 10^{6}$ cells/plate density in $35 \mathrm{~mm}$ Petri dishes previously coated with poly-L-lysine. Regular characterization of the embryonic neuronal cultures confirmed the presence of $95 \%$ neurons in these cultures, as determined by GFAP and NeuN-immunostaining. Primary cultures were kept at $37{ }^{\circ} \mathrm{C}$ in a humidified atmosphere containing $5 \% \mathrm{CO}_{2}$. After 6 days in culture, $50 \mu \mathrm{l}$ of Tf-lipoplexes containing anti-luciferase or nonsilencing (Mut) siRNAs were added to cells to final siRNA concentrations of 50 or $100 \mathrm{nM}$ per well. After a $4 \mathrm{~h}$ incubation (in $5 \% \mathrm{CO}_{2}$, at $37^{\circ} \mathrm{C}$ ) the Neurobasal medium was replaced with fresh medium and the cells were further incubated for different periods of time.

\subsection{Confocal microscopy studies}

In order to study the cellular uptake of siRNAs and Tf-lipoplexes and the role of the Tf-receptor in this process, cortical neurons were incubated for $4 \mathrm{~h}$ at $37{ }^{\circ} \mathrm{C}$ with naked or Tf-lipoplex-associated Cy3-labelled siRNAs, prepared at a 3/2 or 2/1 lipid/siRNA charge ratios. In parallel experiments, the cells were incubated with an excess of free $\mathrm{Tf}\left(10 \mathrm{mg} / \mathrm{ml}\right.$ of growth medium) for $1 \mathrm{~h}$ at $37^{\circ} \mathrm{C}$ before the delivery of Tf-lipoplexes, which were then incubated with the cells at $37^{\circ} \mathrm{C}$ for $4 \mathrm{~h}$ in the presence of the same excess of Tf. The cells were then washed with PBS, stained with the fluorescent DNA-binding dye Hoechst $33342(1 \mu \mathrm{g} / \mathrm{ml})$ and mounted in PBS for immediate analysis by confocal microscopy. Confocal fluorescent laser scanning microscopy was performed by using a Zeiss axiovert system (Zeiss, Jena, Germany) equipped with an UV laser (364 nm), an argon laser (470, 488 and $514 \mathrm{~nm}$ ) and two He/Ne lasers (543 nm and $633 \mathrm{~nm}$ ). Confocal microphotographs were taken at $4 \mathrm{~h}$ and up to $24 \mathrm{~h}$ after incubation of neurons with Cy3-labeled siRNA, using the 60× oil immersion objective. All pictures were obtained at a pinhole setting that resulted in analyses of $1 \mu \mathrm{m}$ slices. Hoechst staining was detected at $364 \mathrm{~nm}$ excitation/385 nm longpass filter emission and Cy3/siGlo was detected by $\mathrm{He} / \mathrm{Ne}$ laser $543 \mathrm{~nm}$ excitation and 560 longpass filter emission.

\subsection{Evaluation of luciferase activity}

Luciferase activity was measured in total protein extracts obtained from in vitro primary cortical cultures, after delivery of Tf-lipoplexes containing anti-luciferase or non-silencing (Mut) siRNAs. For in vivo experiments, total protein extracts were obtained from the dissected cortex, corpus callosum and striatum of both contralateral and ipsilateral brain hemispheres of C57/BL6 transgenic NF-kB luciferase reporter mice, previously injected with Tf-lipoplexes containing anti-luciferase or nonsilencing (Mut) siRNAs. Luciferase activity was measured using a luciferase detection kit, according to the protocol provided by the manufacturer (Promega, Madinson, WI, USA). Briefly, $48 \mathrm{~h}$ after delivery of Tf-lipoplexes the tissue or cells were lysed with luciferase lysis buffer (Promega) for $15 \mathrm{~min}$ at room temperature. Twenty microliters of protein extract were removed from each well and added to $100 \mu \mathrm{l}$ of luciferase assay buffer, containing luciferin. Luciferase activity, measured as relative light units (RLU), was immediately evaluated in a SpectraFluorPlus luminometer (Tecan, Männedorf, Switzerland). Total protein levels in each sample were measured using the BCA protein assay
(Pierce, Rockford, IL, USA) and normalization of luciferase activity levels was performed before statistical analysis. The data were expressed as percentage of control (non-transfected) samples.

\subsection{Evaluation of cell viability and apoptosis}

Cell viability of primary neuronal cultures, following Tf-lipoplex delivery was determined by 3-(4,5dimethylthiazol-2-y1)-2,5-diphenyltetrazolium bromide (MTT) reduction. The MTT assay allows to measure cell viability through evaluation of changes in the cellular mitochondrial metabolic activity. Forty-eight hours after transfection, cells were washed with fresh growth medium and incubated with MTT $(0.25 \mathrm{mg} / \mathrm{ml})$ for $2 \mathrm{~h}$ at $37^{\circ} \mathrm{C}$. The reaction was terminated by adding dimethylsulfoxide solution and the absorbance was determined at $590 \mathrm{~nm}$ and $630 \mathrm{~nm}$ in an ELISA microplate reader (Spectra FluorPlus; Tecan, Durham, NC, USA). Cell viability was calculated as percentage of control cells (non-transfected) using the formula: $\left(A_{590}-A_{630}\right)$ of treated cells $\times 100 /\left(A_{590}-A_{630}\right)$ of control cells.

In parallel experiments, following Tf-lipoplex delivery, cultured neurons were stained with the fluorescent DNA-binding dye Hoechst 33342. Stained nuclei were visualized by epifluorescence microscopy using a Zeiss Axiovert 200 microscope (Zeiss, Jena, Germany), equipped with the $20 \times$ objective. Neurons with condensed or fragmented nuclei were considered apoptotic. Two hundred cells per culture were counted and counts were made in at least six separate cultures per treatment condition without knowledge of treatment history. Results were expressed as percentage of apoptotic cells with respect to control (non-transfected cells).

\subsection{Extraction of RNA and $c D N A$ synthesis}

Twenty-four hours after anti-c-Jun or Mut siRNA delivery, total RNA was extracted from $1 \times 10^{6}$ neuronal cells using the RNeasy Mini Kit (Qiagen, Hilden, Germany), according to the manufacturer's recommendations for cultured cells. Briefly, after cell lysis, the total RNA was adsorbed to a silica matrix, washed with the recommended buffers and eluted with $40 \mu \mathrm{l}$ of RNase-free water by centrifugation. After RNA quantification, cDNA conversion was performed using the Superscript III First Strand Synthesis Kit (Invitrogen, Karlsruhe, Germany), according to the manufacturer's instructions. For each sample, cDNA was produced from $0.5 \mu \mathrm{g}$ of total RNA in a iQ 5 thermocycler (Bio-Rad), by applying the following protocol: $10 \mathrm{~min}$ at $25^{\circ} \mathrm{C}, 30 \mathrm{~min}$ at $55^{\circ} \mathrm{C}$ and 5 min at $85^{\circ} \mathrm{C}$. After cDNA synthesis, a 30 min incubation period with RNase $\mathrm{H}$ at $37^{\circ} \mathrm{C}$ was performed in order to remove any remaining RNA contamination. Finally, the cDNA was diluted 1:3 with RNase-free water prior to quantification by QRT-PCR.

\subsection{Quantitative Real Time Polymerase Chain Reaction (QRT-PCR)}

Quantitative PCR was performed in an iQ 5 thermocycler (Bio-Rad) using 96-well microtitre plates and the iQ SYBR Green Supermix Kit (Bio-Rad). The primers for the target gene (c-Jun) and the two tested house keeping genes (GAPDH and HPRT) were pre-designed by Qiagen (QuantiTect Primer, Qiagen). A master mix was prepared for each primer set, containing a fixed volume of SYBR Green Supermix and the appropriate amount of each primer to yield a final concentration of $150 \mathrm{nM}$. For each reaction, $20 \mu \mathrm{l}$ of master mix were added to $5 \mu \mathrm{l}$ of template cDNA. All reactions were performed in duplicate (two cDNA reactions per RNA sample) at a final volume of $25 \mu \mathrm{l}$ per well, using the iQ5 Optical System Software (Bio-Rad). The reaction conditions consisted of enzyme activation and well-factor determination at $95{ }^{\circ} \mathrm{C}$ for $1 \mathrm{~min}$ and $30 \mathrm{~s}$ followed by 40 cycles at $95{ }^{\circ} \mathrm{C}$ for $10 \mathrm{~s}$ (denaturation), $30 \mathrm{~s}$ at $55^{\circ} \mathrm{C}$ (annealing) and $30 \mathrm{~s}$ at $72{ }^{\circ} \mathrm{C}$ (elongation). The melting curve protocol started immediately after amplification and consisted of $1 \mathrm{~min}$ heating at $55^{\circ} \mathrm{C}$ followed by eighty $10 \mathrm{~s}$ steps, with $0.5^{\circ} \mathrm{C}$ increases in temperature at each step. Threshold values for 
threshold cycle determination $\left(C_{\mathrm{t}}\right)$ were generated automatically by the iQ5 Optical System Software. The percentage of c-Jun knockdown was determined by the $\Delta \Delta C_{\mathrm{t}}$ method, using GAPDH as reference gene,

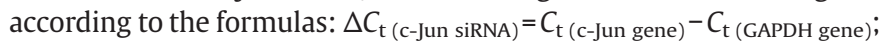

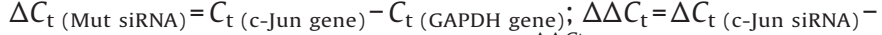
$\Delta C_{\mathrm{t}}$ (Mut siRNA); \% knockdown $=100 \%-\left(2^{-\Delta \Delta C \mathrm{t}} \times 100\right)$. The $\Delta \Delta C_{\mathrm{t}}$ value indicates the changes in RNA transcription caused by treatment with anti-c-Jun siRNAs, normalized to RNA transcription changes in neurons treated with Mut siRNAs. When positive, the $\Delta \Delta C_{\mathrm{t}}$ value indicates downregulation of the target mRNA.

\subsection{Western blot analysis}

Forty-eight or $72 \mathrm{~h}$ after anti-c-Jun or Mut siRNA delivery, protein extracts were obtained from neuronal primary cultures using a lysis buffer containing protease inhibitors (Sigma). Protein content was determined using the Bio-Rad protein quantification kit (Bio-Rad) and $20 \mu \mathrm{g}$ of total protein were resuspended in loading buffer $(20 \%$ glycerol, 10\% SDS, $0.1 \%$ bromophenol blue), incubated for $2 \mathrm{~min}$ at $95{ }^{\circ} \mathrm{C}$ and loaded onto a $10 \%$ polyacrylamide gel. After electrophoresis the proteins were blotted onto a PVDF membrane according to standard protocols. After blocking in 5\% non-fat milk, the membrane was incubated with an anti-c-Jun antibody (1:100) (Cell Signalling, USA) overnight at $4{ }^{\circ} \mathrm{C}$, and with the appropriate secondary antibody (1:20000) (Amersham, Uppsala, Sweden) for $2 \mathrm{~h}$ at room temperature. Equal protein loading was shown by reprobing the membrane with an anti- $\alpha$-tubulin antibody $(1: 10000)$ (Sigma) and with the same secondary antibody. After this incubation period, the blots were washed several times with saline buffer (TBS/T $-25 \mathrm{mM}$ Tris- $\mathrm{HCl}$, $150 \mathrm{mM} \mathrm{NaCl}, 0.1 \%$ Tween and $5 \mathrm{mg} / \mathrm{ml}$ non-fat powder milk) and incubated with ECF (alkaline phosphatase substrate; $20 \mu \mathrm{l}$ of ECF $/ \mathrm{cm}^{2}$ of membrane) for $5 \mathrm{~min}$ at room temperature and then submitted to fluorescence detection at $570 \mathrm{~nm}$ using a VersaDoc Imaging System Model 3000 (Bio-Rad). For each membrane, the analysis of band intensity was performed using the Quantity One software (Bio-Rad).

\subsection{Stereotactic injection of Tf-lipoplexes}

For intrastriatal injections of Tf-lipoplexes or glucose buffer (HBG), C57/BL6 mice were anaesthetized with halothane and placed in a stereotactic apparatus. A midline incision was made, the soft tissues were reflected and a burr hole was made in the skull with the aid of a surgery micro-drill, at a point $0.0 \mathrm{~mm}$ anterior from bregma and $2.5 \mathrm{~mm}$ lateral from the mideline (sagittal suture), according to Paxinos and Watson [42]. A volume of $2 \mu \mathrm{l}$ of HBG or Tf-lipoplexes containing anti-luciferase or non-silencing (Mut) siRNAs were injected at a rate of $0.2 \mu \mathrm{l} / 30 \mathrm{~s}$ in the right hemisphere (ipsilateral hemisphere) of each animal, via a stainless steel needle connected to a Hamilton syringe (Hamilton Bonaduz, Bonaduz, GR, Switzerland), $2.75 \mathrm{~mm}$ ventral from dura. Five minutes after the injection was completed, the needle was withdrawn slowly and the skin was sutured. No symptoms of toxicity or loss of basic activity were observed in any of the mice receiving Tflipoplexes. The animals were sacrificed in deep halothane anaesthesia $48 \mathrm{~h}$ after Tf-lipoplex administration and the brains were removed and placed on an acrylic matrix. A two mm section surrounding the injection site was cut with a stainless steel razor and dissected in order to remove the cortex, corpus callosum and upper half of the striatum of both hemispheres of each animal for analysis of luciferase activity and total protein quantification.

\subsection{Tf-lipoplex distribution in the brain}

In order to study the internalization of Tf-lipoplexes and their in vivo distribution, a group of C57/BL6 mice received Tf-lipoplexes containing Cy3-labelled siRNAs in their ipsilateral hemisphere. The mice were sacrificed under deep halothane anaesthesia $24 \mathrm{~h}$ after transfection and coronal sections from the striatal region $(20 \mu \mathrm{m})$ were cut in a cryostat (Leica CM 3050S, Leica, Wetzlar, Germany) at $-20{ }^{\circ} \mathrm{C}$. The sections were immediately observed by epifluorescence microscopy under a Zeiss Axiovert microscope, equipped with the 20× objective and the rhodamine filter or by confocal microscopy under a Zeiss Axiovert system. Confocal microphotographs were taken using the $60 \times$ oil immersion objective. Cy3/siGlo was detected by $\mathrm{He} / \mathrm{Ne}$ laser $543 \mathrm{~nm}$ excitation and $560 \mathrm{~nm}$ longpass filter emission

\subsection{Histological evaluation of Tf-lipoplex toxicity}

Forty-eight hours after Tf-lipoplex delivery, the mice were deeply anaesthetized with an overdose of avertin $(10 \mu \mathrm{l} / \mathrm{g})$ and transcardially perfused with $20 \mathrm{ml}$ of an ice-cold $0.9 \% \mathrm{NaCl}$ solution, followed by $20 \mathrm{ml}$ of ice-cold $4 \%$ paraformaldehyde in $0.9 \% \mathrm{NaCl}$ solution. The brains were removed and postfixed $(12 \mathrm{~h})$ in the same fixative solution, followed by 2-3 days in a cryoprotection solution containing $25 \%$ sucrose. After this period, the brains were rapidly frozen in dry ice, dipped in OCT embedding medium (Sakura Finetek, Mijdrecht, The Netherlands), and $30 \mu \mathrm{m}$ sections were cut at $-20{ }^{\circ} \mathrm{C}$ in a cryostat (Leica) $1000 \mu \mathrm{m}$ anterior and $1000 \mu \mathrm{m}$ posterior from the injection site. The sections were mounted and dried in gelatine-coated glass slides and each slide was stained for $5 \mathrm{~min}$ in $0.5 \%$ cresyl violet solution (Sigma) in acetate buffer, rinsed twice in water, briefly dehydrated in ethanol, cleared in xylene solution and mounted with Entellan (Merck). The striatal slices were examined under a Zeiss Axiovert microscope, equipped with $5 \times$ and $20 \times$ objectives. Tissue was considered damaged when intensively stained condensed or fragmented nuclei (pycnotic nuclei) were present and only few healthy cells remained.

\subsection{Statistical analysis}

All data are presented as mean \pm standard deviation (SD). In vitro data are the result of three independent experiments each performed at least in triplicate. One Way ANOVA analysis of variance combined with Tukey posthoc test was used for multiple comparisons in cell culture experiments.

In vivo data were analysed by the Kruskal-Wallis One Way Analysis of Variance, followed by the Dunnett's posthoc test for multiple comparisons between groups. Statistical differences are presented at probability levels of $p<0.05, p<0.01$ and $p<0.001$. Calculations were performed with standard statistical software (GraphPad Prism 4).

\section{Results}

\subsection{Cellular uptake of fluorescently labelled siRNAs}

In order to study the in vitro uptake of Tf-lipoplexes by neuronal cells, the internalization and distribution of naked or Tf-lipoplexassociated Cy3-labelled siRNAs were evaluated by confocal microscopy in primary cortical cultures $24 \mathrm{~h}$ following delivery. The results shown in Fig. 1 reveal that at this time point cells treated with Tf-lipoplexes, prepared at $3 / 2$ or $2 / 1$ lipid/siRNA charge ratios (panels a-f), present intense red fluorescence throughout the cytoplasm and nucleus, indicating widespread distribution of labelled siRNAs. In contrast, the delivery of the same amount of naked Cy3siRNAs (panels g-i) did not allow detection of red fluorescence inside the cells or even in the extracellular medium, indicating that naked siRNAs are not efficiently internalized, which may be due to inefficient binding to the cell surface, most likely as a result of washing or RNaserelated degradation.

It has been demonstrated that Tf is highly effective in promoting lipoplex-mediated transfection in various cell lines and primary cell cultures [15-17,26]. Our previous studies have also suggested that Tf enhances cellular uptake of lipoplexes and induces destabilization of 

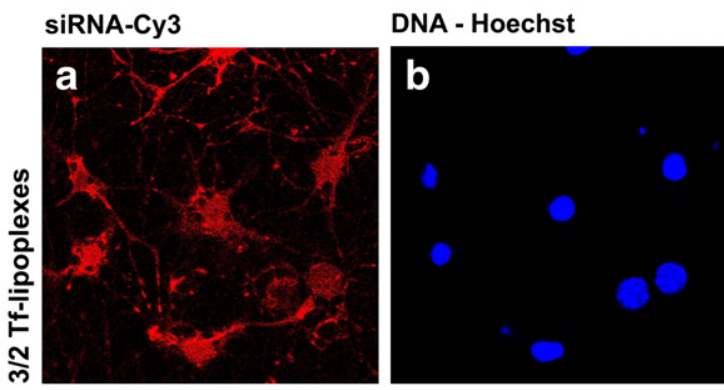

Merge
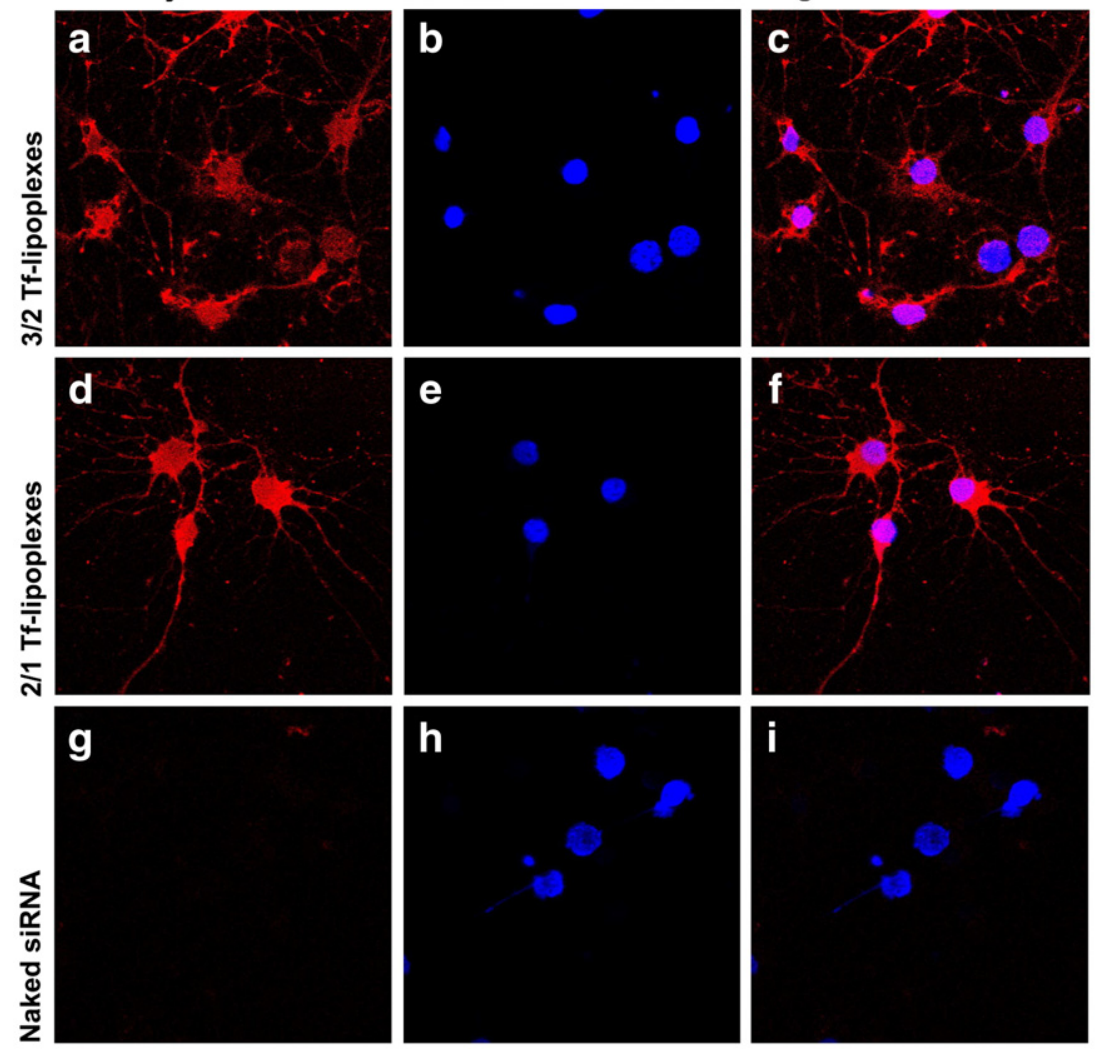

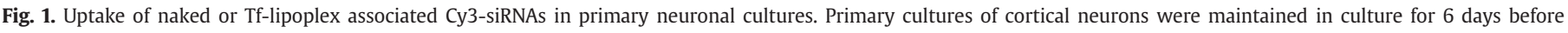

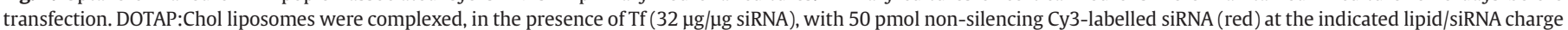

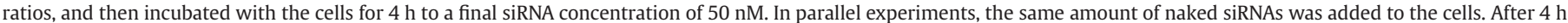

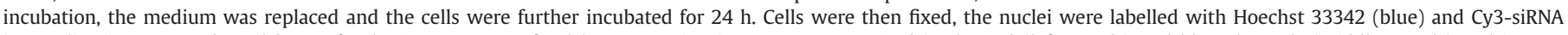

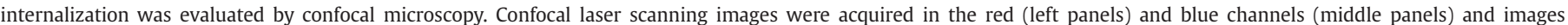
representative of three independent experiments are presented separately and as a merged image (right panels) at 600× magnification.

the endosome membrane, promoting cytoplasmic release of DNA $[19,20]$. Aiming at examining the role of Tf-receptors in siRNA uptake mediated by Tf-lipoplexes, primary neuronal cultures were preincubated in the presence or absence of iron-saturated $\mathrm{Tf}$ (holo-Tf) for $1 \mathrm{~h}$ at $37{ }^{\circ} \mathrm{C}$, before the addition of Tf-lipoplexes containing Cy3labelled siRNAs. Fig. 2 illustrates the effect of pre-incubating neuronal cultures with $10 \mathrm{mg} / \mathrm{ml}$ of free $\mathrm{Tf}$ (approximately 100 times the amount of protein that is associated with the lipid/siRNA complexes) on both Tf-lipoplex internalization (Fig. 2a) and Tf-lipoplex-mediated gene silencing (Fig. 2b). As observed in Fig. 2, the presence of this large excess of Tf during the transfection period reduces the number of fluorescent particles located inside the cells $4 \mathrm{~h}$ after Tf-lipoplex delivery (Fig. 2, panels i and ii). This effect is even more pronounced at $24 \mathrm{~h}$ after delivery, where a significant decrease in intracellular fluorescence is observed in cells incubated with excess of Tf (Fig. 2, panels iii and iv). Nevertheless, a low intensity of red fluorescence can still be observed, suggesting that under this condition a small degree of internalization did still occur. In order to study the effect of the excess of free Tf on transfection efficiency, parallel experiments were performed in neurons treated with Tf-lipoplexes prepared at the 3/2 and 2/1 lipid/siRNA charge ratios and containing anti-luciferase siRNAs (Fig. 2b). Luciferase activity was measured $48 \mathrm{~h}$ after transfection. The decrease in luciferase activity reached $50 \%$ for both charge ratios in the absence of excess Tf (normal medium), compared with only a $15 \%$ and $25 \%$ reduction for the $3 / 2$ and $2 / 1$ lipid/siRNA charge ratios, respectively, in the presence of excess Tf. Altogether, these results indicate that Tf-lipoplex internalization is highly dependent on the availability of Tf-receptors on the cell surface, although non-specific cell interactions also contribute to transfection efficiency.

\subsection{Gene silencing efficiency and biocompatibility of Tf-lipoplexes}

To assess the silencing efficiency in vitro mediated by Tf-lipoplexes and to exclude possible off-target effects caused by the vector per se or by the transfection process, the levels of luciferase gene expression were measured in luciferase-expressing primary neuronal cultures, $48 \mathrm{~h}$ after delivery of anti-luciferase (Luc) or non-silencing (Mut) siRNAs (Fig. 3). Parallel transfection experiments employing Lipofectamine 2000, which is frequently used for in vitro DNA and siRNA delivery, were performed for comparison. As shown in Fig. 3, both lipoplex formulations (3/2 and 2/1 lipid/siRNA charge ratios) achieved approximately $50 \%$ reduction in luciferase activity, when prepared with Luc siRNAs, a result which is slightly higher than that obtained with the standard reagent - Lipofectamine 2000. Gene silencing was found to be specific, since no significant decrease in luciferase levels was observed for both formulations with Mut siRNAs at concentrations of $50 \mathrm{nM}$. It should be noted that the increase in siRNA concentration to $100 \mathrm{nM}$ resulted in a slight improvement in luciferase knockdown but was also accompanied by non-specific silencing, as shown by the decrease of luciferase activity obtained for this concentration when employing Mut siRNAs. This finding demonstrated that optimization of the siRNA concentration is always necessary to achieve both efficient and specific silencing effects.

Postmitotic neuronal cells are more susceptible to cationic lipidinduced toxicity than most cell lines and other primary cultures of 


\section{a Normal Tf}

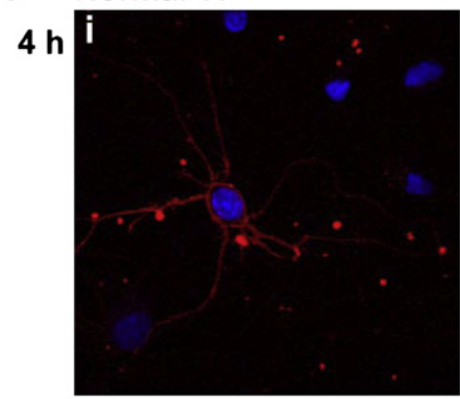

Excess Tf

$24 \mathrm{~h}$

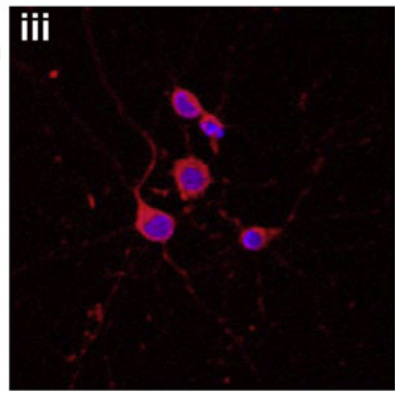

b

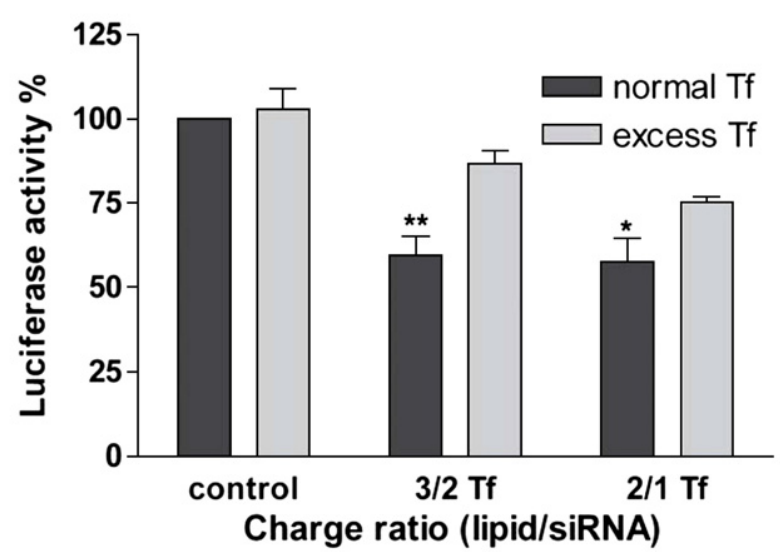

Fig. 2. Effect of excess of transferrin on Tf-lipoplex uptake and transfection efficiency in primary neuronal cultures. Primary cultures of cortical neurons were maintained in culture for 6 days before transfection. DOTAP:Chol liposomes were complexed, in the presence of Tf ( $32 \mu \mathrm{g} / \mu \mathrm{g}$ siRNA), with $50 \mathrm{pmol}$ of (a) non-silencing Cy3-labelled siRNAs (red) or (b) anti-luciferase siRNAs and added to the cells to a final siRNA concentration of $50 \mathrm{nM}$. (a) Cells were incubated with Tf-lipoplexes prepared at a 3/2 lipid/siRNA charge ratio, for $4 \mathrm{~h}$ in the absence of a pre-treatment with $\mathrm{Tf}$ (panels $\mathrm{i}$ and iii) or in medium supplemented with excess of $\operatorname{Tf}(10 \mathrm{mg} / \mathrm{ml}$ ) (panels ii and iv). Four or $24 \mathrm{~h}$ after transfection, cells were fixed and the nuclei labelled with Hoechst 33342 (blue). The extent of Cy3-siRNA internalization was evaluated by confocal microscopy. Representative confocal laser scanning images are presented as merged images at 600× magnification. Experiments were performed at least three times. (b) Cells were incubated for $4 \mathrm{~h}$ with Tf-lipoplexes at the indicated charge ratios. After this period the medium was replaced and the cells were further incubated for $48 \mathrm{~h}$ before evaluation of transfection efficiency. Luciferase activity was measured in each sample as relative light units (RLU) and calculated as percentage of activity in control (non-transfected) cells. Mean RLU \pm S.D. was obtained from three independent experiments, each performed in triplicate. ${ }^{*} p<0.05$ and ${ }^{* *} p<0.01$ compared to cells transfected without pre-treatment with excess Tf.

mitotic cells. To evaluate whether Tf-lipoplexes are toxic to neuronal cultures in vitro, cell viability was assessed by the MTT assay $48 \mathrm{~h}$ after transfection. Results presented in Fig. 4a show that no significant change in cell viability was observed after delivery of Tf-lipoplexes, independently of the employed lipid/siRNA charge ratio, when the final siRNA concentration was $50 \mathrm{nM}$. Nevertheless, Tf-lipoplexes prepared for a final siRNA concentration of $100 \mathrm{nM}$, presented a slight toxic effect leading to approximately $20 \%$ decrease in cell viability, which strongly correlates with the non-specific effect observed for Tf- lipoplexes at high siRNA concentrations (Fig. 3). In parallel studies, the number of pycnotic nuclei was evaluated in neuronal cultures $48 \mathrm{~h}$ after Tf-lipoplex delivery. As shown in Fig. 4b, no significant increase in the number of condensed nuclei was observed after transfection in the presence of $50 \mathrm{nM}$ siRNAs, whereas a two-fold increase was obtained following delivery of $100 \mathrm{nM}$ siRNAs, which is consistent with the results from the MTT assay. On the other hand, neurons transfected with $50 \mathrm{nM}$ siRNA complexed with Lipofectamine showed a three-fold increase in the number of cells with abnormal nuclear morphology.

\subsection{Tf-lipoplex-mediated c-Jun mRNA and protein knockdown in primary neuronal cultures}

In order to demonstrate that Tf-lipoplex ability to promote efficient gene silencing is not restricted to specific reporter targets, such as luciferase, an endogenous gene was selected to further investigate Tflipoplex transfection properties. The well described transcription factor (c-Jun), whose role in the apoptotic process is currently being investigated, was chosen as target and a complementary siRNA was synthesized based on the mouse c-Jun mRNA sequence. To establish the average decrease in c-Jun mRNA levels (Table 1), cortical neurons were incubated with Tf-lipoplexes prepared at the 3/2 lipid/siRNA charge ratio and containing anti-c-Jun or non-functional Mut siRNAs. Twenty-four hours after Tf-lipoplex delivery, c-Jun mRNA silencing was evaluated using QRT-PCR. The percentage of c-Jun knockdown in these cells was calculated using the $\Delta \Delta C_{\mathrm{t}}$ method, as described in the Materials and methods section. In these experiments, the mean $\Delta \Delta C_{t}$ value found was 1.22 , which translates into $54 \%$ of c-Jun knockdown. Moreover, two endogenous reference genes, whose expression should not differ between samples, were used in this study as normalization controls. The expression levels of both GAPDH and HPRT genes did not

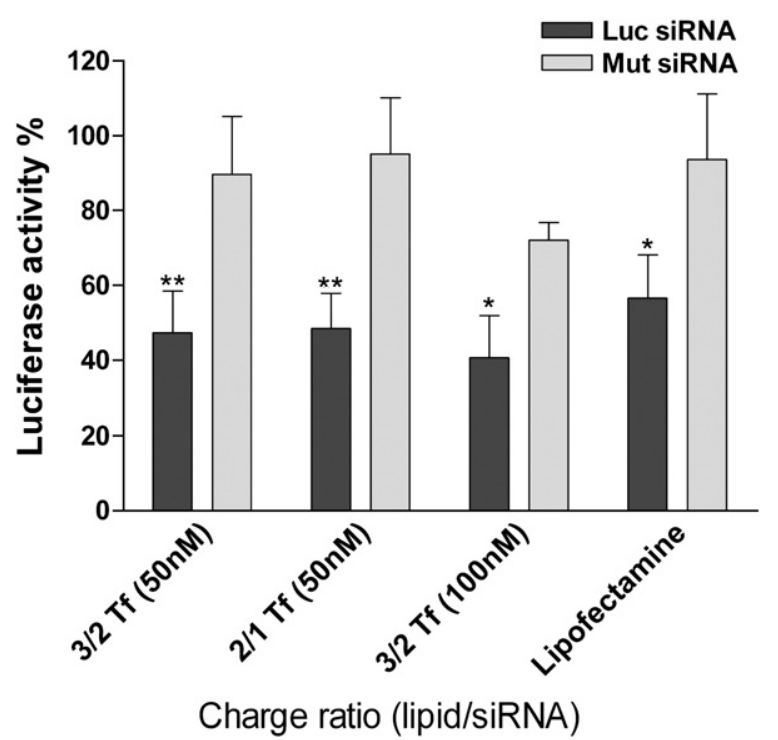

Fig. 3. In vitro gene silencing mediated by Tf-lipoplexes. Primary cultures of cortical neurons were maintained in culture for 6 days before transfection. DOTAP:Chol liposomes were complexed, in the presence of Tf ( $32 \mu \mathrm{g} / \mu \mathrm{g}$ siRNA), with $50 \mathrm{pmol}$ or 100 pmol of anti-Luciferase (Luc) or non-silencing (Mut) siRNA at the indicated lipid/ siRNA charge ratios, and then added to the cells at a final siRNA concentration of $50 \mathrm{nM}$ or $100 \mathrm{nM}$, respectively. In parallel experiments, cortical neurons were incubated with $50 \mathrm{pmol}$ of anti-Luciferase (Luc) or non-silencing (Mut) siRNAs complexed with Lipofectamine at a final siRNA concentration of $50 \mathrm{nM}$. After $4 \mathrm{~h}$ incubation, the medium was replaced and the cells were further incubated for $48 \mathrm{~h}$. Luciferase activity was measured in each sample as relative light units (RLU) and calculated as percentage of activity in control (non-transfected) cells. Mean RLU \pm S.D. was obtained from three independent experiments, each performed in triplicate. ${ }^{*} p<0.05$ and ${ }^{* *} p<0.01$ compared to cells treated with Mut siRNA. 
a

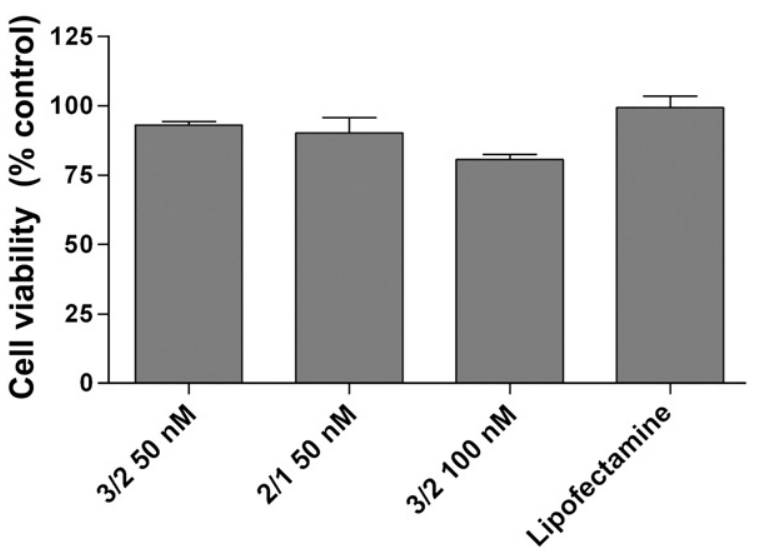

Charge ratio (lipid/siRNA)

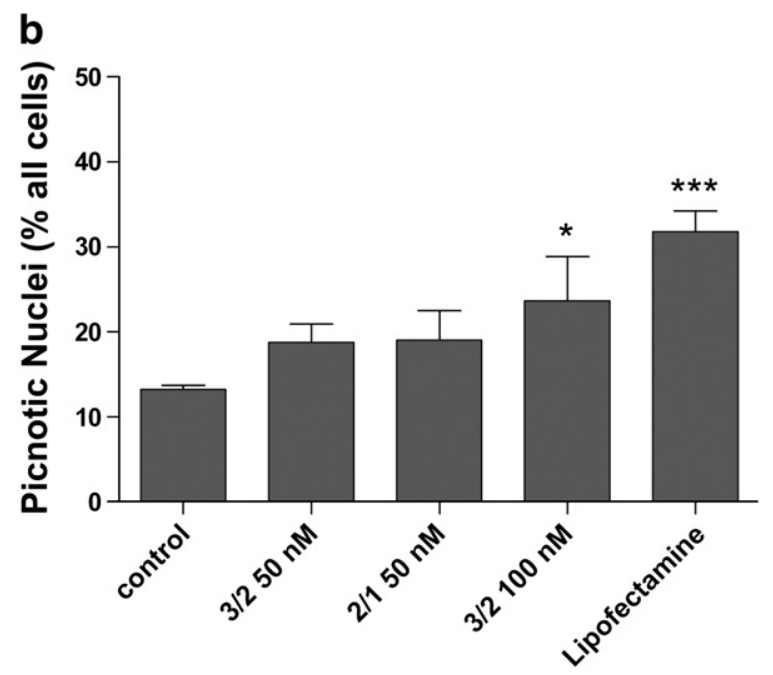

Charge ratio (lipid/siRNA)

Fig. 4. Effect of Tf-lipoplexes on the viability of primary neuronal cultures. Primary cultures of cortical neurons were maintained in culture for 6 days before transfection. DOTAP:Chol liposomes were complexed, in the presence of Tf (32 $\mu \mathrm{g} / \mu \mathrm{g}$ siRNA), with $50 \mathrm{pmol}$ or $100 \mathrm{pmol}$ of siRNA at the indicated lipid/siRNA charge ratios, and then added to the cells to a final siRNA concentration of $50 \mathrm{nM}$ or $100 \mathrm{nM}$, respectively. In parallel experiments, cortical neurons were incubated with $50 \mathrm{pmol}$ of non-silencing (Mut) siRNAs complexed with Lipofectamine at a final siRNA concentration of $50 \mathrm{nM}$. After $4 \mathrm{~h}$ incubation, the medium was replaced and the cells were further incubated for $48 \mathrm{~h}$. (a) Cell viability was determined by the MTT assay. The data are expressed as percentage of control (non-transfected) cells. (b) Apoptotic cells were visualized after labelling of nuclei with Hoechst 33342. For each condition, the number of pycnotic nuclei is expressed as the percentage of total number of nuclei. Results were obtained from three independent experiments, each performed in triplicate. ${ }^{*} p<0.05$ and ${ }^{* * *} p<0.001$ compared to control (non-treated) neurons.

differ between control (non-transfected) cells and cells treated with Tf-complexes, which indicates that no unspecific mRNA downregulation occurs attributable to the vector per se.

The efficiency of siRNA-mediated gene silencing was confirmed by Western blot analysis. In parallel experiments, neurons exposed to a similar transfection protocol were collected and lysed 48 or $72 \mathrm{~h}$ following Tf-lipoplex delivery. Fig. 5a illustrates a pronounced protein knockdown, obtained after Tf-lipoplex-mediated delivery of anti-cJun siRNAs. The protein quantification analysis (Fig. 5b) performed 48 and $72 \mathrm{~h}$ after siRNA delivery, shows similar results for both tested time points, indicating approximately $45 \%$ decrease in c-Jun levels with respect to control. These results were found to be specific, since no relevant c-Jun knockdown was observed with Mut siRNA and the levels of $\alpha$-tubulin were constant for all tested formulations.
Table 1

QRT-PCR quantification of Tf-lipoplex-mediated c-Jun knockdown in neuronal cells

\begin{tabular}{lllllll}
\hline Neuronal cells & $C_{\mathrm{t}}$ & $C_{\mathrm{t}}$ & $\Delta C_{\mathrm{t}}$ & $\Delta \Delta C_{\mathrm{t}}$ & $\begin{array}{l}\text { c-Jun } \\
\text { knockdown (\%) }\end{array}$ & $\begin{array}{l}\text { Average c-Jun } \\
\text { knockdown (\%) }\end{array}$ \\
\hline Transfected with & 34.93 & 20.62 & 14.31 & 1.08 & $\mathbf{5 3}$ & \\
anti-c-Jun siRNA & 32.90 & 20.90 & 12.00 & 0.6 & $\mathbf{3 4}$ & $\mathbf{5 4}$ \\
& 35.21 & 20.10 & 15.10 & 1.98 & $\mathbf{7 5}$ & \\
Transfected with & 34.21 & 20.98 & 13.23 & & & \\
Mut siRNA & 31.90 & 20.50 & 11.40 & & & \\
& 33.37 & 20.25 & 13.12 & & & \\
\hline
\end{tabular}

The levels of both c-Jun and HPRT genes were determined by quantitative RT-PCR $24 \mathrm{~h}$ after transfection. c-Jun knockdown was evaluated by the $\Delta \Delta C_{\mathrm{t}}$ method $(n=3)$ using the GAPDH gene as reference gene. Results are expressed as a percentage of c-Jun expression in cells treated with Mut siRNAs.

\subsection{In vivo Tf-lipoplex-mediated delivery of Cy3-labelled siRNAs}

We have previously demonstrated that association of $\mathrm{Tf}$ to lipoplexes promoted efficient delivery of DNA in vivo [16]. Therefore, in this work we evaluated whether this strategy would also result in the enhancement of siRNA delivery to the mouse brain. For this purpose, C57/BL6 mice were injected with Tf-lipoplexes prepared at a $2 / 1 \mathrm{lipid} /$ siRNA charge ratio and containing $3 \mu \mathrm{g}$ of Cy3-labelled siRNAs. The injection was performed in the striatum of the right hemisphere (ipsilateral hemisphere) of each animal and observation of the neuronal tissue of these animals was made $24 \mathrm{~h}$ after Tf-lipoplex delivery. Fig. 6a provides evidence that stereotactic injection of Tflipoplexes leads to efficient siRNA delivery in vivo, as observed by the high number of red-labelled cells in the ispsilateral hemisphere (Fig. 6a, panel i) and the intracellular localization of the Cy3-siRNAs observed by confocal microscopy (Fig. 6a, panels iii and iv) in z-stack

a

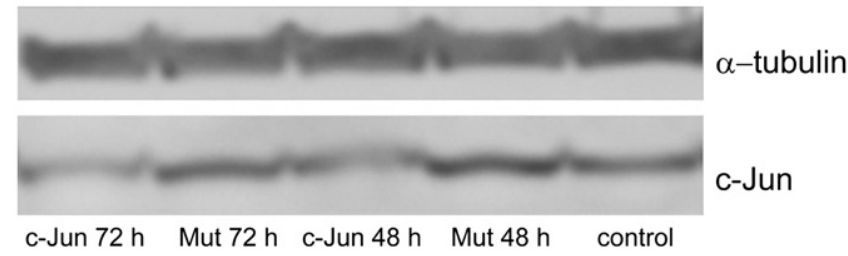

b

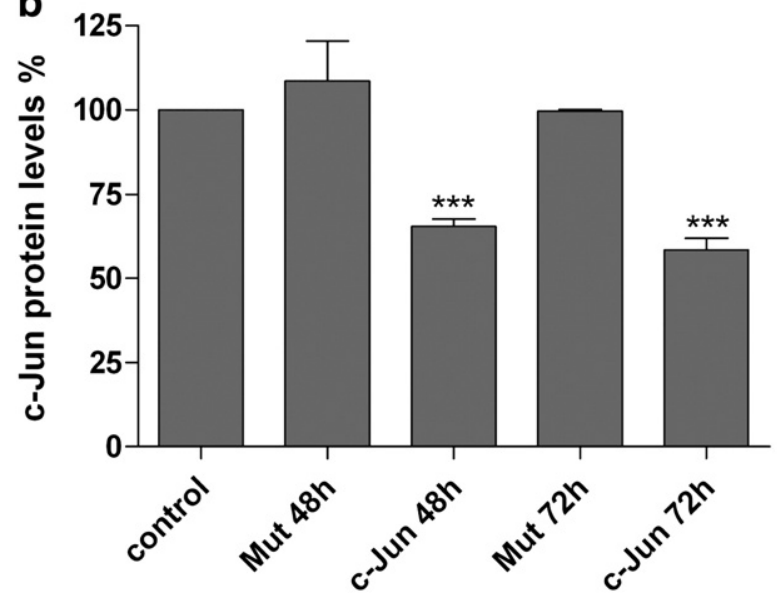

Fig. 5. Western blot quantification of Tf-lipoplex-mediated c-Jun knockdown in neurons. Primary cultures of cortical neurons were maintained in culture for 6 days before transfection. DOTAP:Chol liposomes were complexed, in the presence of Tf $(32 \mu \mathrm{g} / \mu \mathrm{g}$ siRNA), with $50 \mathrm{pmol}$ of siRNA at the $3 / 2(+/-)$ lipid/siRNA charge ratio, and then added to the cells to a final siRNA concentration of $50 \mathrm{nM}$. After $4 \mathrm{~h}$ incubation, the medium was replaced and the cells were further incubated for $48 \mathrm{~h}$ or $72 \mathrm{~h}$ before c-Jun and $\alpha$-tubulin quantification by immunoblotting. (a) Representative gel showing c-Jun knockdown. (b) Quantification of c-Jun silencing observed in (a), corrected for individual $\alpha$-tubulin signal intensity. Results are expressed as percentage of c-Jun expression levels with respect to untreated controls. ${ }^{* * *} p<0.001$ compared to control (non-treated) neurons. 
slices of this hemisphere. As expected, no red labelling was detected in the contralateral hemisphere (Fig. 6a, panel ii). In all injected animals the red fluorescence was distributed throughout the entire superior region of the striatum, mostly pronounced in the area surrounding the injection site, but was not observed in other adjacent brain regions such as the cortex or corpus callosum.

In order to evaluate Tf-lipoplex biocompatibility, counterstaining of brain slices with cresyl violet was performed. No relevant signs of toxicity were found throughout the ipsilateral striatum where, similarly to observations in the contralateral hemisphere, neurons presented a light violet colour and large cell body characteristic of healthy cells (Fig. $6 \mathrm{~b})$. The presence of vacuolization or large number of apoptotic cells was not detected in any of the injected animals and only a small number of pycnotic cells were observed in the tissue surrounding the injection site. These dark coloured and shrunken cells were present in the slices showing the injection tract, and are a common consequence of tissue damage occurring during the insertion of the injection needle.

\subsection{In vivo luciferase silencing mediated by Tf-lipoplexes}

In order to evaluate the in vivo gene silencing activity of Tflipoplexes, transgenic Nf-kB luciferase reporter mice were injected in

\section{a}
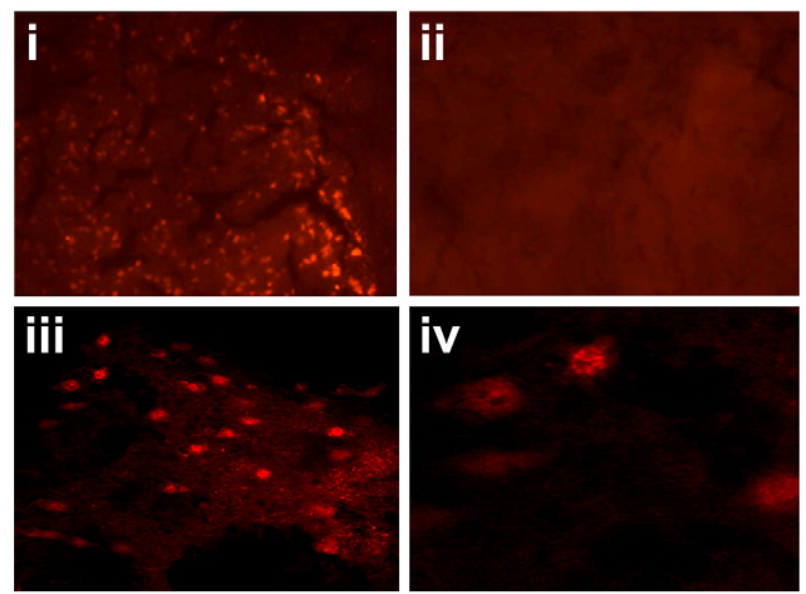

b
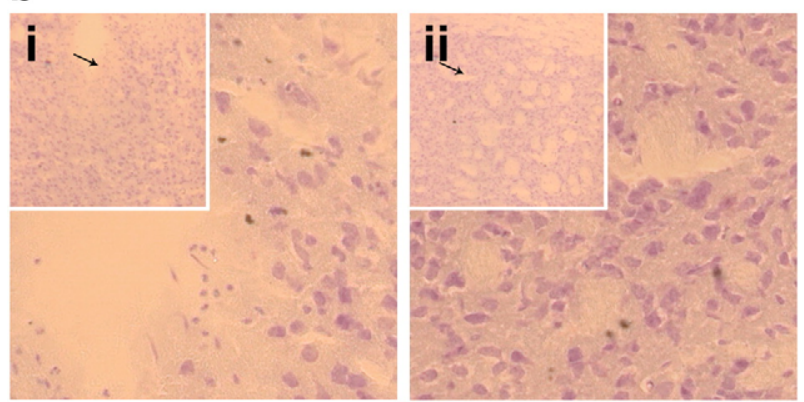

Fig. 6. Tf-lipoplex-mediated internalization and biocompatibility of Cy3-labelled siRNAs in vivo. Animals were injected stereotactically, in the striatum of the right (ipsilateral) hemisphere, with Tf-lipoplexes containing $3 \mu \mathrm{g}$ of Cy3-labelled siRNAs and prepared at the 2/1 lipid/siRNA charge ratio; no injection was performed in the left (contralateral hemisphere). Animals were sacrificed $24 \mathrm{~h}$ postinjection and (a) Tf-lipoplex-mediated internalization of Cy3-siRNAs was evaluated in $30 \mu \mathrm{m}$ coronal brain sections by fluorescence microscopy (panels i and ii) or confocal microscopy (panels iii and iv). Biocompatibility of Tf-lipoplexes was evaluated in adjoining sections by cresyl violet staining (b). (a) Representative fluorescence microscopy images of (i) ipsilateral and (ii) contralateral hemispheres are presented at 1000× magnification. Representative $z$ stack images of the same brain region in the ipsilateral hemisphere are presented at a $6000 \times$ magnification (iii). A specific area of each $z$-stack image was zoomed $2 \times$ to obtain close-up images showing intracellular localization of Cy3-siRNAs (iv). (b) Representative microscopy images of (i) ipsilateral and (ii) contralateral hemispheres are presented at both $2000 \times$ and 500× magnifications. The black arrows indicate the region further magnified.

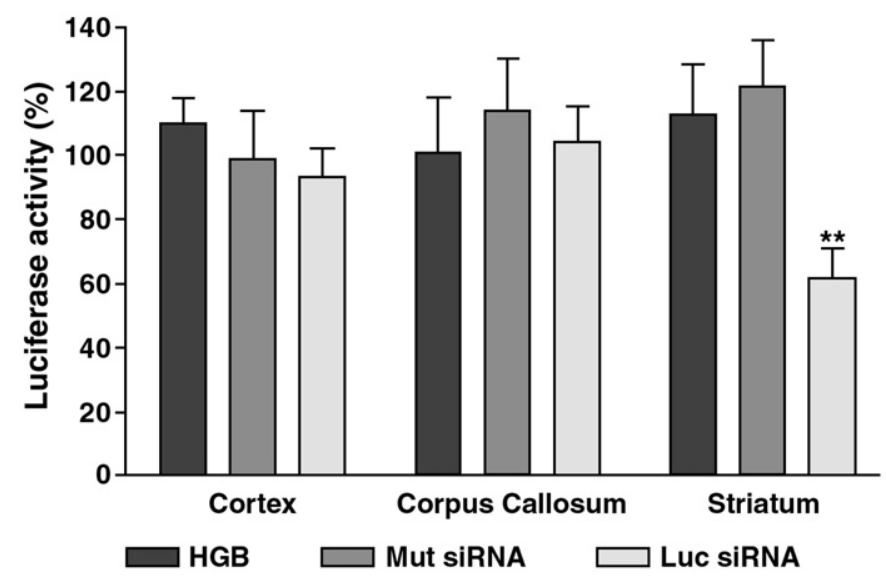

Fig. 7. In vivo luciferase silencing mediated by Tf-lipoplexes. Animals were injected stereotactically, in the striatum of the right (ipsilateral) hemisphere, with Tf-lipoplexes containing $3 \mu \mathrm{g}$ of anti-luciferase (Luc) or non-silencing (Mut) siRNAs, prepared at the 2/1 lipid/siRNA charge ratio in a volume of $2 \mu \mathrm{l}$, or with an equal volume of vehicle (HBG); no injection was performed in the left (contralateral) hemisphere. Animals were sacrificed $48 \mathrm{~h}$ postinjection and luciferase activity was evaluated in the striatum, corpus callosum and cortex of both hemispheres of each animal. Luciferase activity was measured in each tissue sample as relative light units (RLU) and calculated as percentage of activity with respect to the contralateral (non-injected) hemisphere, after normalization to total protein levels. Results are expressed as mean RLU \pm SD obtained from $n=5$ animals per group. ${ }^{* *} p<0.01$ compared to striatum luciferase levels of both HBG and Mut siRNA-treated animals.

the striatum of the right (ipsilateral) hemisphere with $3 \mu \mathrm{g}$ of Luc or Mut siRNAs complexed with Tf-containing DOTAP:Chol liposomes, in a final injection volume of $2 \mu$ l. In parallel experiments, a third group of animals received an injection of HBG buffer $(2 \mu \mathrm{l})$ which was used for Tf-lipoplex preparation. Fig. 7 presents the levels of luciferase activity observed in the ipsilateral upper striatum and adjacent regions (cortex and corpus callosum) of each animal group $48 \mathrm{~h}$ after Tf-lipoplex delivery. Results are expressed as the percentage of luciferase activity compared to the non-injected (contralateral) hemispheres. The Luc siRNA-treated group presented a significant decrease (approximately 40\%) of luciferase levels in the ispsilateral striatal tissue, whereas no luciferase knockdown was detected in the ipsilateral cortex or corpus callosum of these animals. The fact that no decrease in luciferase activity was observed after transfection with Mut siRNAs indicates that Tf-lipoplex-mediated gene silencing in vivo does not present detectable unspecific effects when low siRNA and lipid concentrations are used. Also, no significant increase in luciferase expression was detected in the ipsilateral hemispheres of the animals treated with Mut siRNAs or HBG, suggesting that the injection per se did not induce any unspecific NF-kB activation.

Altogether these results clearly show that Tf-lipoplexes are able to mediate efficient siRNA delivery and gene silencing, both in neuronal primary cultures and in the mouse brain, with high specificity and minimum toxicity.

\section{Discussion}

In this work we provide evidence that Tf-lipoplexes, a cationic lipid-based formulation, previously employed in our recent work for siRNA delivery in vitro [15] and DNA delivery to neuronal cells in vitro [17] and in vivo [16], can efficiently mediate siRNA delivery into neuronal primary cultures, using low amounts of siRNA and low lipid/ siRNA charge ratios. Confocal microscopy revealed that both charge ratios tested (3/2 and $2 / 1 \mathrm{lipid} /$ siRNA) led to the intracellular localization of Cy3-siRNAs $24 \mathrm{~h}$ after delivery (Fig. 1, panels a-f). In contrast, after incubation with naked Cy3-labelled siRNAs, no fluorescence was observed inside or outside the neuronal cells (Fig. 1, panels g-i). These results are very similar to those obtained 
in cell lines [15] and represent clear evidence of insufficient cellular uptake of naked siRNAs, that is likely attributable to nucleasemediated degradation and low binding affinity to the cell membrane. The data also suggest that Tf-lipoplexes increase significantly the rate of siRNA internalization in neuronal cells, probably by protecting the nucleic acids from nuclease-mediated degradation and by enhancing siRNA uptake through the endocytic pathway.

In order to clarify the actual mechanisms of Tf-lipoplex-mediated siRNA delivery and to evaluate the contribution of Tf-receptors in this process, we examined Cy3-siRNA internalization in the presence of an excess of holo-transferrin (100 times more than the amount used for Tf-lipoplex preparation) at 4 and $24 \mathrm{~h}$ after transfection. Results in Fig. 2a show that Tf-lipoplex internalization is partly inhibited in the presence of large amounts of Tf, as judged by the low intracellular fluorescence levels observed for both incubation times (Fig. 2, panels ii and iv), as compared to those found in the absence of any excess of Tf (Fig. 2, panels i and iii). The fact that excess Tf does not completely inhibit Cy3-siRNA uptake suggests that the cell internalization of Tflipoplexes containing siRNAs is mediated by both specific, i.e. Tfreceptor-dependent, and non-specific, Tf-receptor-independent processes. This partial unspecificity was confirmed by the results obtained with the luciferase assay (Fig. 2b), after delivery of Tflipoplexes containing anti-luciferase (Luc) siRNAs. These studies show that pre-saturation of Tf-receptors with excess of free Tf leads to a decrease in gene silencing mediated by Tf-lipoplexes prepared at a 3/2 lipid/siRNA charge ratio, as compared to cells transfected in normal medium, while gene silencing mediated by Tf-lipoplexes prepared at a $2 / 1$ lipid/siRNA charge ratio was only slightly affected. The observation that the inhibition of transfection is less significant for the 2/1 lipid/ siRNA charge ratio might be explained by the excess positive charge in this formulation, which can promote non-specific receptor-mediated uptake of the Tf-lipoplexes, in a similar way to what was previously observed for DNA-containing Tf-lipoplexes. This effect can be attributed to the establishment of pronounced electrostatic interactions between the negatively charged cell membrane and the positive charges of the cationic lipid, which promote Tf-lipoplex binding to the cell surface.

Taken together, the results presented in Fig. 2 suggest that Tflipoplex internalization is mediated by both Tf-receptor-dependent and -independent mechanisms. The Tf-receptor-independent mechanism, which seems to be the most relevant to successful gene silencing, presents a strong dependence on the presence of an excess of positive charges. These results partly correlate with those from previous work on the cellular uptake of DNA-containing Tf-lipoplexes, in which it is clearly shown that Tf-lipoplex internalization is mostly independent of the Tf-receptors[19]. Here, we show that gene silencing is only slightly affected by the presence of excess Tf, especially when Tf-lipoplexes are prepared at a high charge ratio. Although in our previous work, no role was attributed to Tf-receptors in Tf-lipoplex internalization, in the present study a small percentage of Tf-lipoplexes carrying siRNA seems to be internalized and mediate a gene silencing effect through Tf-receptors, which may be explained by small differences in the structure of the complexes. In both cases, transferrin was electrostatically associated to the cationic liposomes, prior to complexation with DNA or siRNA. In the case of DNA-containing Tf-lipoplexes, it is possible that the transferrin binding motifs are not available at the surface of the lipoplexes to bind to its receptors on the target cells. On the other hand, in the case of siRNA-containing Tf-lipoplexes, the small size of siRNA molecules may allow exposure of some of the Tf binding moieties, allowing the binding of Tf-lipoplexes to transferrin receptors.

In order to evaluate the biological activity and gene silencing potential of Tf-lipoplexes in vitro, we performed transfection experiments employing Luc or non-silencing (Mut) siRNAs in neuronal primary cultures obtained from luciferase-expressing transgenic mice. A large body of evidence including our own work suggests that there are significant differences in gene silencing efficiency when comparing siRNA protocols in transiently transfected cells with the same siRNA application in stably transfected cells or when targeting endogenous protein expression. Usually, siRNA appears to be very potent when applied as a co-transfection with the targeted reporter gene in transiently transfected cells. However, the same protocol is far less efficient when targeting the same reporter gene in stably transfected cells. Therefore stably transfected cells present better models than transiently expressing cells and should be preferentially used in gene silencing studies. The transgenic model (expression of single genomic copy driven by a NF-kB responsive promoter), employed throughout this work, is even a better approach considering that luciferase is expressed like an endogenous gene in these mice. In fact, we have demonstrated this when transferring according siRNA protocols for luciferase silencing to applications in wildtype neurons and neuronal cell lines where we successfully silenced endogenous expression of c-Jun, Bid or AIF [15,41].

The results presented in Fig. 3 clearly demonstrate that Tflipoplexes can mediate an efficient downregulation of the luciferase gene with concomitant decrease in luciferase activity (50\%) for a siRNA concentration of $50 \mathrm{nM}$. Our observations also suggest that this is the ideal siRNA concentration to achieve highly specific gene knockdown in neuronal cultures. As illustrated in Fig. 3, the increase of siRNA concentration to $100 \mathrm{nM}$, although slightly improving gene silencing, enhanced the downregulation of the luciferase gene by the Mut sequence which is a clear indicator of non-specific effects. This result once again stresses the importance of always using the lowest effective dose of both siRNA and delivery agent in order to reach a balance between efficiency and specificity, minimizing non-specific protein decrease and thereby reducing the risk of an immune reaction, mediated through Toll-like receptor activation [43-45].

The in vitro toxicity assays (Fig. 4) revealed that cell viability was not affected upon transfection with Tf-lipoplexes prepared at low siRNA concentrations for both charge ratios tested. Also, no significant increase in the number of apoptotic cells was observed for these formulations when compared to control (non-transfected) cultures. The increase of siRNA concentration to $100 \mathrm{nM}$ induced a decrease in cell viability of approximately $20 \%$, and an increase of $10 \%$ in the number of pycnotic nuclei. Notably, even at this siRNA concentration, Tf-lipoplexes presented better biocompatibility than Lipofectamine 2000 , which increased the percentage of apoptotic cells to $20 \%$ when complexed with $50 \mathrm{nM}$ siRNA.

In order to further elucidate the potential of Tf-lipoplexes to mediate gene silencing of endogenous genes, and in line with previous results obtained in neuronal cell lines [15], we selected the c-Jun gene as target and investigated c-Jun knockdown in neuronal primary cultures. The QRT-PCR results presented in Table 1 show a significant downregulation of c-Jun mRNA (54\%) 24 h after delivery of Tfcomplexes, without detectable off-target effects. Moreover, following Western blot analysis, a noteworthy reduction of c-Jun protein levels (45\%) was also observed in these cells (Fig. 5), Due to its important role as a transcription factor in several intracellular apoptotic pathways, cJun is being currently studied as a promising target for the treatment of neurodegenerative conditions and excitotoxic brain injury (manuscript in preparation). These results clearly show the ability of Tflipoplexes to mediate gene silencing of endogenous targets, such as cJun, which illustrates the potential of this formulation to help both in the clarification of the molecular mechanisms involved in this type of neuronal cell death and in the increase of cell survival following Tflipoplex-mediated target silencing.

Once overcoming the first obstacles for achieving gene silencing in neuronal cultures, which mainly concern the efficient uptake of intact siRNA molecules and their release into the cytoplasm, the in vivo application of siRNAs to the brain still faces major challenges due to the poor accessibility of this organ and its complex physiology. We have previously shown that DOTAP:Chol liposomes associated with 
transferrin are able to promote pDNA delivery to the neuronal tissue in vivo following local injection [16]. In the present work, we investigated whether similar results would be observed for Tf-lipoplex-mediated siRNA delivery. In order to study in vivo siRNA uptake, Cy3-labelled siRNAs were injected into the mouse striatum and the distribution of intracellular fluorescence was observed $24 \mathrm{~h}$ after transfection by both fluorescence and confocal microscopy. Our results indicate that the tested formulation is able to mediate delivery of siRNA molecules in an extensive area surrounding the injection site (Fig. 6a) without any signs of neuronal toxicity, as observed by cresyl violet counterstaining (Fig. 6b). Z-stack confocal images (Fig. 6a, panels iii and iv) also show that Cy3-siRNAs are efficiently internalized by neurons following Tflipoplex delivery. Results from parallel experiments employing Cy3labelled siRNAs naked or formulated in lipoplexes in the absence of transferrin (plain lipoplexes) showed that no siRNA uptake was detected in the case of naked Cy3-siRNAs, and only a small number of cells close to the injection site showed red labelling when plain lipoplexes were used (data not shown). These data further illustrate the role of transferrin in enhancing lipoplex uptake in neuronal cells. Studies performed in transgenic animals stably expressing luciferase clearly demonstrated that significant downregulation of a reporter protein could be achieved through Tf-lipoplex-mediated gene silencing. The results presented in Fig. 7 indicate a specific siRNA-induced decrease in luciferase activity in the striatum. Approximately $40 \%$ of such decrease was confined to this brain region and was only detected after anti-luciferase siRNA delivery, clearly demonstrating that the observed gene silencing effect is attributed to the selected siRNA sequence and not to non-specific effects of the vector per se. It is also important to note that no significant increase in luciferase expression was detected in the ipsilateral hemispheres of the animals treated with Mut siRNAs or HBG, with respect to contralateral hemispheres. A significant advantage of the NF-kB luciferase reporter system is the capability to indicate adverse effects that may be caused by siRNA applications. For example, potential immunogenic or inflammatory reactions after local siRNA application would result in a significant NF-kB activation and thus increased luciferase activity, easily detectable in the different treatment groups. Toxic effects resulting in a general inhibition of protein synthesis or cell death would likewise be easily detected in animals receiving the vehicle or non-functional control siRNA. The data presented in Figs. 6 and 7 clearly demonstrate that local application of the siRNA lipoplexes did not cause any of these potential adverse effects besides the desired specific silencing of luciferase.

It should be stressed that although $40 \%$ gene downregulation does not lead to full protein knockdown, it will be sufficient to promote a significant therapeutic effect, which may be particularly important in certain cases where the complete abolishment of a given protein could exert deleterious effects to the cells. This is case when the target protein presents multiple, even contradictory, physiological roles. The transcription factor c-Jun is one of such proteins, which in normal conditions regulates the expression of multiple essential proteins and, therefore, is vital per se to cell survival, but can also be strongly involved in pro-apoptotic mechanisms following injury.

\section{Conclusion}

Overall, our results demonstrate that, similarly to what was previously shown for plasmid DNA, Tf-lipoplexes are able to promote siRNA transfection in neuronal cells, both in vitro and in vivo, with high efficiency and minimum toxicity, which clearly shows their potential regarding the downregulation of neurological diseaserelated genes for future therapeutic application.

\section{Acknowledgements}

A.L.C. Cardoso is the recipient of a fellowship from the Portuguese Foundation for Science and Technology (SFRH/BD/17216/2004). This work was partially financed by a grant from the Portuguese Foundation for Science and Technology (PTDC/BIO/65627/2006). C. Culmsee is supported by the Alzheimer Forschungs Initiative. The authors thank Emma J. Esser for careful proof reading of the manuscript.

\section{References}

[1] M.A. Hossain, Molecular mediators of hypoxic-ischemic injury and implications for epilepsy in the developing brain, Epilepsy Behav. 7 (2) (2005) 204-213.

[2] A.K. Liou, R.S. Clark, D.C. Henshall, X.M. Yin, J. Chen, To die or not to die for neurons in ischemia, traumatic brain injury and epilepsy: a review on the stress-activated signaling pathways and apoptotic pathways, Prog. Neurobiol. 69 (2) (2003) 103-142.

[3] S.L. Mehta, N. Manhas, R. Raghubir, Molecular targets in cerebral ischemia for developing novel therapeutics, Brain Res. Rev. 54 (1) (2007) 34-66.

[4] S. Genc, T.F. Koroglu, K. Genc, RNA interference in neuroscience, Brain Res. Mol Brain Res. 132 (2) (2004) 260-270.

[5] D.W. Sah, Therapeutic potential of RNA interference for neurological disorders, Life Sci. 79 (19) (2006) 1773-1780

[6] F.Y. Xie, M.C. Woodle, P.Y. Lu, Harnessing in vivo siRNA delivery for drug discovery and therapeutic development, Drug Discov. Today 11 (1-2) (2006) 67-73.

[7] N.J. Caplen, J.P. Taylor, V.S. Statham, F. Tanaka, A. Fire, R.A. Morgan, Rescue of polyglutamine-mediated cytotoxicity by double-stranded RNA-mediated RNA interference, Hum. Mol. Genet. 11 (2) (2002) 175-184

[8] V.M. Miller, H. Xia, G.L. Marrs, C.M. Gouvion, G. Lee, B.L. Davidson, H.L. Paulson, Allelespecific silencing of dominant disease genes, Proc. Natl. Acad. Sci. U. S. A. 100 (12) (2003) 7195-7200.

[9] G. Liu, F. Wong-Staal, Q.X. Li, Development of new RNAi therapeutics, Histol Histopathol. 22 (2) (2007) 211-217.

[10] F. Czauderna, M. Fechtner, S. Dames, H. Aygun, A. Klippel, G.J. Pronk, K. Giese, J. Kaufmann, Structural variations and stabilising modifications of synthetic siRNAs in mammalian cells, Nucleic Acids Res. 31 (11) (2003) 2705-2716.

[11] A.D. Judge, G. Bola, A.C. Lee, I. MacLachlan, Design of noninflammatory synthetic siRNA mediating potent gene silencing in vivo, Mol. Ther. 13 (3) (2006) 494-505.

[12] K. Kariko, M. Buckstein, H. Ni, D. Weissman, Suppression of RNA recognition by Toll-like receptors: the impact of nucleoside modification and the evolutionary origin of RNA, Immunity 23 (2) (2005) 165-175.

[13] Z. Paroo, D.R. Corey, Challenges for RNAi in vivo, Trends Biotechnol. 22 (8) (2004) 390-394.

[14] A. Santel, M. Aleku, O. Keil, J. Endruschat, V. Esche, G. Fisch, S. Dames, K. Loffler, M Fechtner, W. Arnold, K. Giese, A. Klippel, J. Kaufmann, A novel siRNA-lipoplex technology for RNA interference in the mouse vascular endothelium, Gene Ther.13 (16) (2006) 1222-1234.

[15] A.L. Cardoso, S. Simoes, L.P. de Almeida, J. Pelisek, C. Culmsee, E. Wagner, M.C. Pedroso de Lima, siRNA delivery by a transferrin-associated lipid-based vector: a non-viral strategy to mediate gene silencing, J. Gene Med. 9 (3) (2007) 170-183.

[16] M.T. da Cruz, A.L. Cardoso, L.P. de Almeida, S. Simoes, M.C. de Lima, Tf-lipoplexmediated NGF gene transfer to the CNS: neuronal protection and recovery in an excitotoxic model of brain injury, Gene Ther. 12 (16) (2005) 1242-1252.

[17] M.T. da Cruz, S. Simoes, M.C. de Lima, Improving lipoplex-mediated gene transfer into C6 glioma cells and primary neurons, Exp. Neurol. 187 (1) (2004) 65-75.

[18] D. Simberg, A. Weiss, Y. Barenholz, Reversible mode of binding of serum proteins to DOTAP/cholesterol Lipoplexes: a possible explanation for intravenous lipofection efficiency, Hum. Gene Ther. 16 (9) (2005) 1087-1096.

[19] S. Simoes, V. Slepushkin, P. Pires, R. Gaspar, M.P. de Lima, N. Duzgunes, Mechanisms of gene transfer mediated by lipoplexes associated with targeting ligands or pH-sensitive peptides, Gene Ther. 6 (11) (1999) 1798-1807.

[20] M.T. da Cruz, S. Simoes, P.P. Pires, S. Nir, M.C. de Lima, Kinetic analysis of the initial steps involved in lipoplex-cell interactions: effect of various factors that influence transfection activity, Biochim. Biophys. Acta 1510 (1-2) (2001) 136-151.

[21] K. Crook, B.J. Stevenson, M. Dubouchet, D.J. Porteous, Inclusion of cholesterol in DOTAP transfection complexes increases the delivery of DNA to cells in vitro in the presence of serum, Gene Ther. 5 (1) (1998) 137-143.

[22] D. Hirsch-Lerner, M. Zhang, H. Eliyahu, M.E. Ferrari, C.J. Wheeler, Y. Barenholz, Effect of "helper lipid" on lipoplex electrostatics, Biochim. Biophys. Acta 1714 (2) (2005) 71-84.

[23] D. Simberg, S. Weisman, Y. Talmon, Y. Barenholz, DOTAP (and other cationic lipids): chemistry, biophysics, and transfection, Crit. Rev. Ther. Drug Carr. Syst. 21 (4) (2004) 257-317.

[24] S. Simoes, A. Filipe, H. Faneca, M. Mano, N. Penacho, N. Duzgunes, M.P. de Lima, Cationic liposomes for gene delivery, Expert Opin. Drug Deliv. 2 (2) (2005) 237-254.

[25] S. Simoes, P. Pires, N. Duzgunes, M.C. Pedrosa de Lima, Cationic liposomes as gene transfer vectors: barriers to successful application in gene therapy, Curr. Opin. Mol. Ther. 1 (2) (1999) 147-157.

[26] S. Simoes, V. Slepushkin, E. Pretzer, P. Dazin, R. Gaspar, M.C. Pedroso de Lima, N. Duzgunes, Transfection of human macrophages by lipoplexes via the combined use of transferrin and pH-sensitive peptides, J. Leukoc. Biol. 65 (2) (1999) 270-279.

[27] H. Faneca, S. Simoes, M.C. Pedroso de Lima, Association of albumin or protamine to lipoplexes: enhancement of transfection and resistance to serum, J. Gene Med. 6(6) (2004) 681-692.

[28] H. Li, Z.M. Qian, Transferrin/transferrin receptor-mediated drug delivery, Med. Res. Rev. 22 (3) (2002) 225-250.

[29] N. Shi, R.J. Boado, W.M. Pardridge, Receptor-mediated gene targeting to tissues in vivo following intravenous administration of pegylated immunoliposomes, Pharm. Res. 18 (8) (2001) 1091-1095. 
[30] N. Shi, Y. Zhang, C. Zhu, R.J. Boado, W.M. Pardridge, Brain-specific expression of an exogenous gene after i.v. administration, Proc. Natl. Acad. Sci. U. S. A. 98 (22) (2001) 12754-12759.

[31] H. Hatakeyama, H. Akita, K. Maruyama, T. Suhara, H. Harashima, Factors governing the in vivo tissue uptake of transferrin-coupled polyethylene glycol liposomes in vivo, Int. J. Pharm. 281 (1-2) (2004) 25-33.

[32] P.W. Cheng, Receptor ligand-facilitated gene transfer: enhancement of liposomemediated gene transfer and expression by transferrin, Hum. Gene Ther. 7 (3) (1996) 275-282.

[33] M.C. Pedroso de Lima, S. Simoes, P. Pires, H. Faneca, N. Duzgunes, Cationic lipidDNA complexes in gene delivery: from biophysics to biological applications, Adv. Drug Deliv. Rev. 47 (2-3) (2001) 277-294.

[34] C. Tros de Ilarduya, N. Duzgunes, Efficient gene transfer by transferrin lipoplexes in the presence of serum, Biochim. Biophys. Acta 1463 (2) (2000) 333-342.

[35] S. Simoes, V. Slepushkin, R. Gaspar, M.C. de Lima, N. Duzgunes, Gene delivery by negatively charged ternary complexes of DNA, cationic liposomes and transferrin or fusigenic peptides, Gene Ther. 5 (7) (1998) 955-964.

[36] C. Culmsee, J. Siewe, V. Junker, M. Retiounskaia, S. Schwarz, S. Camandola, S. ElMetainy, H. Behnke, M.P. Mattson, J. Krieglstein, Reciprocal inhibition of p53 and nuclear factor-kappaB transcriptional activities determines cell survival or death in neurons, J. Neurosci. 23 (24) (2003) 8586-8595.

[37] I. Millet, R.J. Phillips, R.S. Sherwin, S. Ghosh, R.E. Voll, R.A. Flavell, A. Vignery, M. Rincon, Inhibition of NF-kappaB activity and enhancement of apoptosis by the neuropeptide calcitonin gene-related peptide, J. Biol. Chem. 275 (20) (2000) 15114-15121.

[38] R.E. Voll, E. Jimi, R.J. Phillips, D.F. Barber, M. Rincon, A.C. Hayday, R.A. Flavell, S. Ghosh, NF-kappa B activation by the pre-T cell receptor serves as a selective survival signal in T lymphocyte development, Immunity 13 (5) (2000) 677-689.

[39] N. Plesnila, L. von Baumgarten, M. Retiounskaia, D. Engel, A. Ardeshiri, R. Zimmermann, F. Hoffmann, S. Landshamer, E. Wagner, C. Culmsee, Delayed neuronal death after brain trauma involves p53-dependent inhibition of NFkappaB transcriptional activity, Cell Death Differ. 14 (8) (2007) 1529-1541.

[40] M.J. Campbell, Lipofection reagents prepared by a simple ethanol injection technique, BioTechniques 18 (6) (1995) 1027-1032.

[41] C. Culmsee, C. Zhu, S. Landshamer, B. Becattini, E. Wagner, M. Pellecchia, K. Blomgren, N. Plesnila, Apoptosis-inducing factor triggered by poly(ADP-ribose) polymerase and Bid mediates neuronal cell death after oxygen-glucose deprivation and focal cerebral ischemia, J. Neurosci. 25 (44) (2005) 10262-10272.

[42] G.F. Paxinos, K.B.J., The Mouse Brain in Stereotaxic Coordinates, Elsevier Academic Press, San Diego, 2004.

[43] J.T. Marques, B.R. Williams, Activation of the mammalian immune system by siRNAs, Nat. Biotechnol. 23 (11) (2005) 1399-1405.

[44] M. Sioud, Innate sensing of self and non-self RNAs by Toll-like receptors, Trends Mol. Med. 12 (4) (2006) 167-176.

[45] C.A. Sledz, M. Holko, M.J. de Veer, R.H. Silverman, B.R. Williams, Activation of the interferon system by short-interfering RNAs, Nat. Cell Biol. 5 (9) (2003) 834-839. 